\title{
A QSAR model of benzoxazole derivatives as potential inhibitors for inosine 5'-monophosphate dehydrogenase from Cryptosporidium paroum
}

\author{
Pratibha Teotia, Surya Prakash Dwivedi \& Neeraja Dwivedi*
}

School of Biotechnology, IFTM University, Moradabad, Utter Predesh, India; Neeraja Dwivedi - Email: neer.miet@gmail.com, *Corresponding author

\begin{abstract}
:
Cryptosporidium parvum is the common enteric protozoan pathogen causing cryptosporidiosis in human. Available drugs to treat cryptosporidiosis are ineffective and there is yet no vaccine against C. parvum. Therefore, it is of interest to design an improved yet effective drug against $C$. parvum. Here, we docked benzoxazole derivatives (collected from literature) with inosine 5monophosphate dehydrogenase (IMPDH) from Cryptosporidium parvum using the program AutoDock 4.2. The docked protein inhibitor complex structure was optimized using molecular dynamics simulation for 5 ps with the CHARMM-22 force field using NAMD (NAnoscale Molecular Dynamics program) incorporated in visual molecular dynamics (VMD 1.9.2) and then evaluating the stability of complex structure by calculating RMSD values. NAMD is a parallel, object-oriented molecular dynamics code designed for high-performance simulation of large biomolecular systems. A quantitative structure activity relationship (QSAR) model was built using energy-based descriptors as independent variable and $\mathrm{pIC}_{50}$ value as dependent variable of fifteen known benzoxazole derivatives with C. parvum IMPDH protein, yielding correlation coefficient $\mathrm{r}^{2}$ of 0.7948 . The predictive performance of QSAR model was assessed using different cross-validation procedures. Our results suggest that a ligand-receptor binding interaction for inosine 5-monophosphate dehydrogenase using a QSAR model is promising approach to design more potent inosine 5-monophosphate dehydrogenase inhibitors prior to their synthesis.
\end{abstract}

Keywords: Cryptosporidium parvum; docking; inosine 5`-monophosphate dehydrogenase; AutoDock 4.2; benzoxazole derivatives.

\section{Background:}

Cryptosporidiosis is the most common food and waterborne diseases with worldwide spread, acting as a common cause of diarrhoea in animals and man [1]. Among the five common Cryptosporidium species in humans, Cryptosporidium paroum (C. parvum) and Cryptosporidium hominis (C. hominis) are responsible for more than $90 \%$ of human cases of cryptosporidiosis [2]. Cryptosporidium is one of the most important parasitic diarrheal disease among young children in developing nations, and is problematic as an opportunistic coinfection with HIV due to increased morbidity and mortality [3, 4]. Currently available drugs are not effective for treating cryptosporidiosis and vaccine therapy is lacking, so new drugs are needed. The sequencing of the genomes of Cryptosporidium parvum revealed a highly streamlined anabolic metabolism with potential choke points that might be exploited in drug design [5]. One such vulnerability lies in the pathway that supplies purine nucleotides for the synthesis of DNA and RNA. Like all protozoan parasites, Cryptosporidium is incapable of de novo purine synthesis and relies on salvage of purines from the host [5]. Adenosine is converted into guanine nucleotides in a streamlined pathway that relies on inosine 5'-monophosphate dehydrogenase (IMPDH) catalyzing the conversion of inosine 5 -monophosphate (IMP) to xanthosine 5`-monophosphate (XMP) [6]. 
In this study, we docked experimentally verified 15 benzoxazole-based inhibitors having inhibitory value $\mathrm{IC}_{50}$ in nM with C. paroum IMPDH using AutoDock 4.2, which resulted in energy-based descriptors. Molecular dynamics (MD) simulation studies of inhibitor - protein complex were performed and after that, we have built quantitative structure activity relationship (QSAR) model using Multiple Linear Regression.

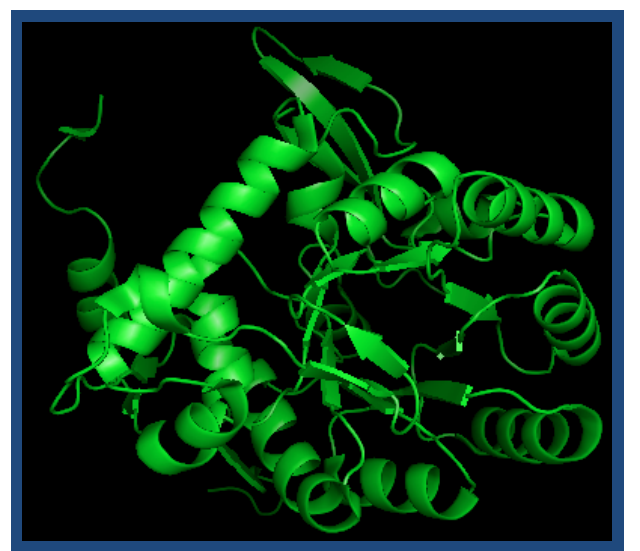

Figure 1: Crystal structure of the catalytic domain of the inosine monophosphate dehydrogenase from cryptosporidium parvum.

\section{Methodology:}

\section{Protein target structure}

The 3D coordinates of the crystal structure of the catalytic domain of the Inosine 5-monophosphate dehydrogenase from cryptosporidium parvum (PDB Id: 4IXH) was retrieved from Protein Databank (http://www.rcsb.org/) and is shown in Figure 1. This is used as a target model for flexible docking. The structure was optimized using the chimera tool [7].

\section{Inhibitors dataset}

Fifteen benzoxazole derivatives with known pIC50 were obtained from Gorla et al. (2013) [8]. The 3D structures of known 15 inhibitors were downloaded in sdf format from pubchem compound database. They were later converted in .pdb format with the help of open babel [9] tool. All the ligands were subjected to energy minimization using the HyperChem software [10].

\section{Molecular docking}

Docking of fifteen benzoxazole derivatives screened from literature against $C$. parvum IMPDH structure were done using molecular docking program AutoDock [11]. Gasteiger charges are added to the ligand and maximum 6 numbers of active torsions are given to the lead compounds using AutoDock tool [12]. Kollman charges and the solvation term were added to the protein structure. The Lamarckian genetic algorithm implemented in Autodock was used for docking.

\section{Molecular dynamics simulations}

Molecular dynamics simulations were done using the NAMD (NAnoscale Molecular Dynamics program; v2.7) graphical interface module [13] incorporated visual molecular dynamics (VMD 1.9.2) [14]. The protein-ligand complex was immersed in the center of a $50 \AA$ box of water molecules where all water molecule atoms (H-O-H) were closer than $1.5 \AA$ and a CHARMM (Chemistry at HARvard Macromolecular Mechanics) 22 parameter file for proteins and lipids; phi and psi cross-term map correction were used in the force field for complexes. For the minimization and equilibration of complex in the water box, we assumed force-field parameters excluding scaling of $1.0 \AA$ and a cutoff of Coulomb forces with a switching function starting at $12 \AA$, reaching zero at a distance of $10 \AA$, ending at $14 \AA$ with a margin of $3.0 \AA$, and all atoms, including those of hydrogen, were illustrated explicitly. A protein structure file (psf) stores structural information of the protein, such as various types of bonding interactions. The psf was created from the initial pdb and topology files using psfgen package of VMD. After running psfgen,two new files were generated protein pdb and protein psf and by accessing PSF and PDB files; NAMD generated the trajectory DCD file. After the simulations, the results were analyzed in VMD by calculating the Root mean square deviation (RMSD) of the complex using rmsd tcl source file from the Tk console and finally rmsd.dat was saved and accessed in Microsoft office excel 2007.

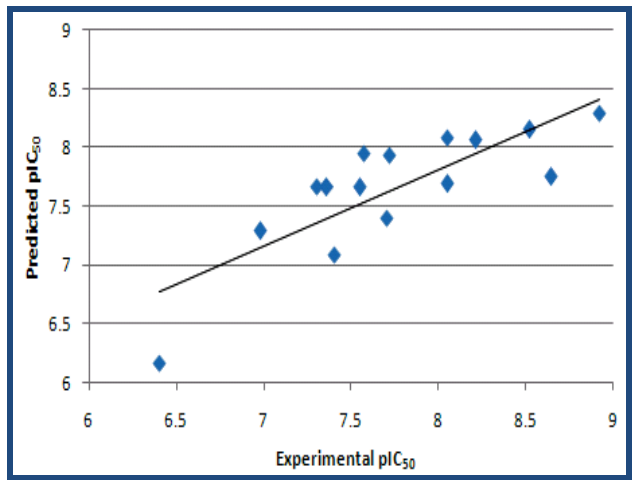

Figure 2: Relationship between experimental ( $x$-axis) and predicted (y-axis) pIC50 values with an $\mathrm{r}^{2}$ value 0.7948 is shown in a QSAR model developed using multiple linear regression analysis.

\section{D QSAR}

A QSAR based model was generated having correlation coefficient $\mathrm{r}^{2}$ value 0.7948 was developed using multiple linear regression analysis. An equation was developed for the inhibitory activities represented as $\mathrm{pIC}_{50}$ values using the six types of energy values as variable descriptors such as Binding Energy (BE), Intermolecular Energy (IME), Internal Energy (IE), Torsional Energy (TorE), vdW + Hbond + desolv Energy (VdwE) and electrostatic energy (EE). A correlation coefficient $\left(\mathrm{r}^{2}\right)$ of 0.7948 was obtained for 15 benzoxazole derivatives as shown below in equation 1 .

Predicted pIC $_{50}=1.0291-26.7693(\mathrm{BE})+25.9634(\mathrm{IME})+$ 0.7866 (IE) + 25.9788 (TorE) $-0.0536(\mathrm{VdwE})-1.7919(\mathrm{EE})$

Several cross-validation procedures were adopted to assess the predictive performance of the QSAR model. In leave-one-out strategy (LOOCV), one molecule was removed from the dataset as a test compound and the remaining 14 molecules were used to build the model. This process was repeated 15 times with each inhibitor as a test molecule. 


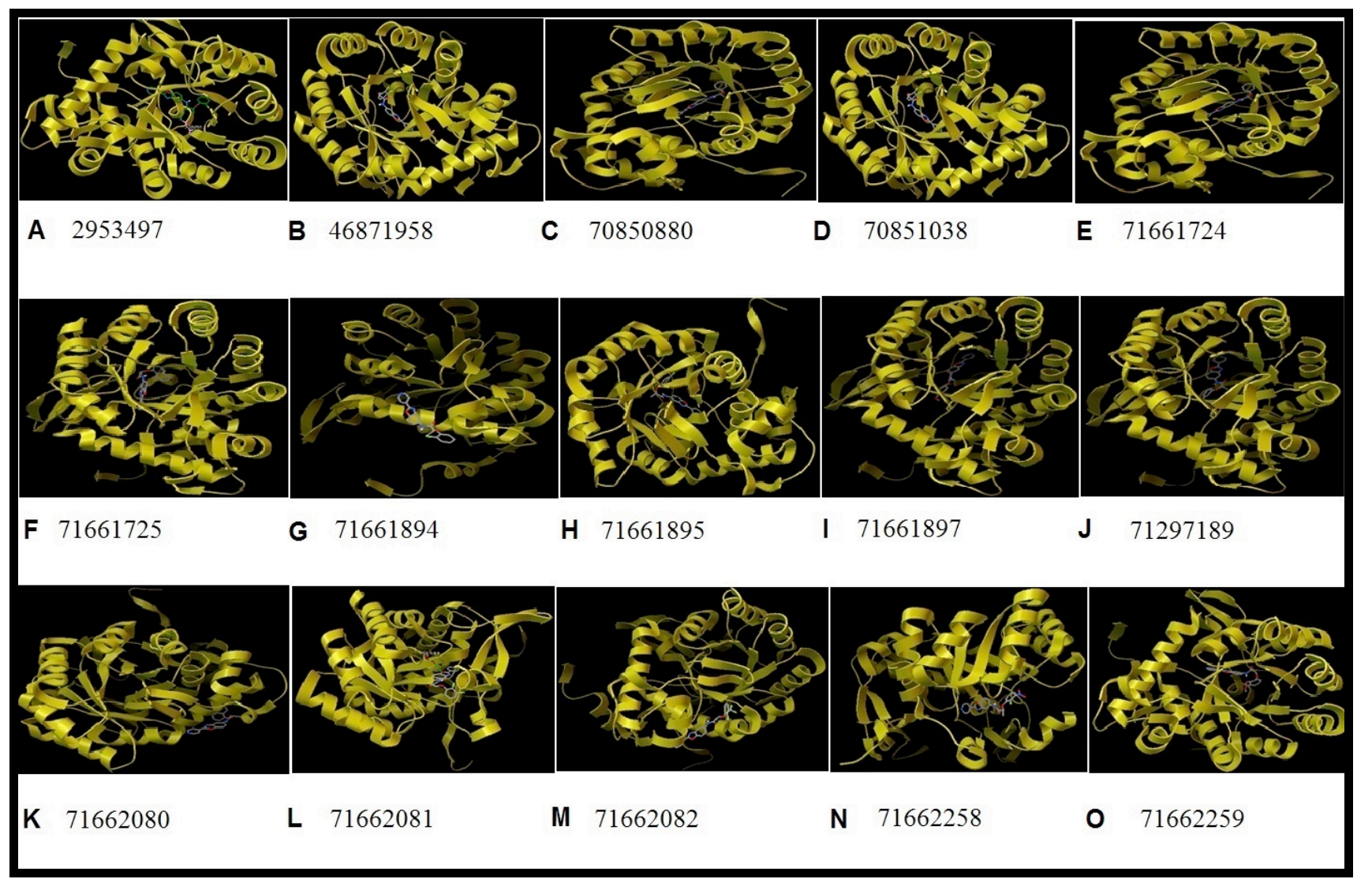

Figure 3: Docking orientation of compounds with IMPDH protein.

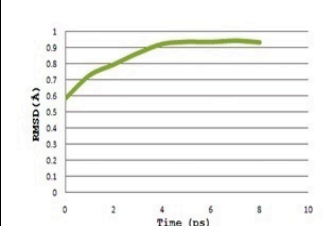

A 2953497

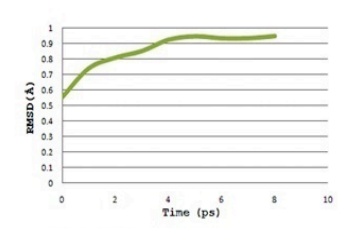

F 71661725

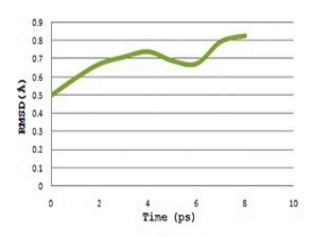

K 71662080

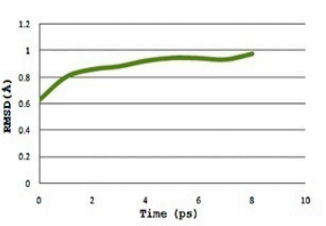

B 46871958

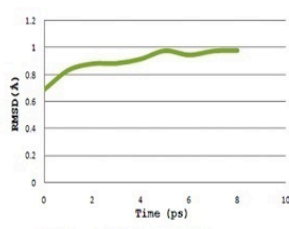

G 71661894

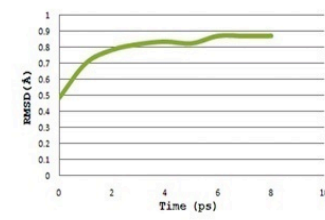

L $\quad 71662081$

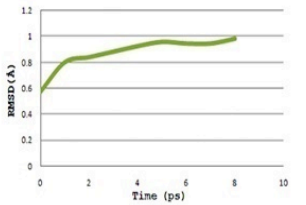

C 70850880

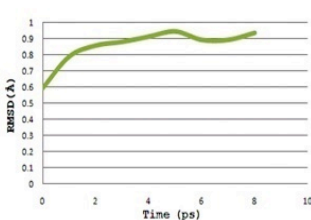

H 71661895

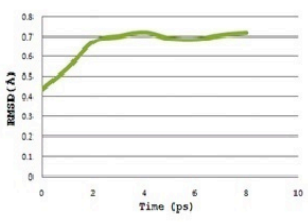

M 71662082

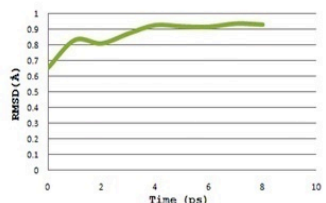

D 70851038

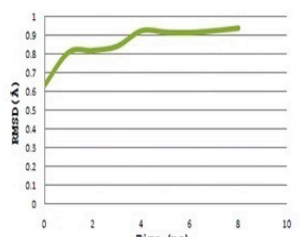

I 71661897

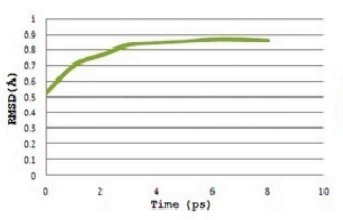

N 71662258

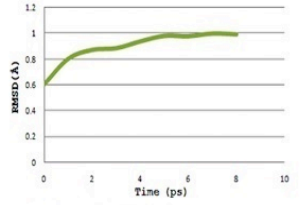

E 71661724

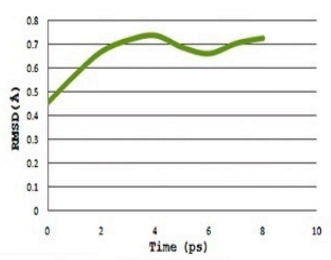

J 71297189

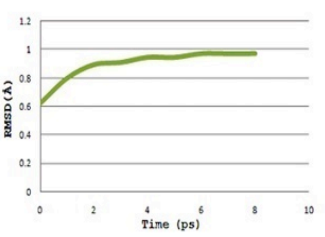

○ 71662259

Figure 4: Graph displaying root mean square deviation (RMSD) of compounds - protein complex versus time (5 ps) at $310 \mathrm{~K}$, resulted in highest peak at $0.98 \AA$. 
Table 1: Benzoxazole derivatives of Cryptosporidium parvum inosine 5'-monophosphate dehydrogenase on the basis of different $\mathrm{R}_{1}$ group.

\begin{tabular}{llll}
\hline & & \\
\hline Sl. No. & PubChem CID & R1 & \\
1 & 2953497 & $2,4-d i-C l P h$ & 7.36 \\
2 & 71661724 & 2-ClPh & 7.72 \\
3 & 71661725 & 4-ClPh & 6.98 \\
4 & 70850880 & Ph & 7.40 \\
5 & 71661894 & 4-OMePh & 7.55 \\
6 & 71661895 & 3-ClPh & 7.70 \\
7 & 46871958 & 2,3-di-ClPh & 8.52 \\
8 & 70851038 & 1-naphthyl & 8.05 \\
9 & 71661897 & 1-(4-Cl-naphthyl) & 7.57 \\
\hline
\end{tabular}

Table 2: Benzoxazole derivatives of Cryptosporidium parvum inosine 5 '-monophosphate dehydrogenase on the basis of different $\mathrm{R}_{1}$ and $\mathrm{X}$ group. Benzoxazole derivatives

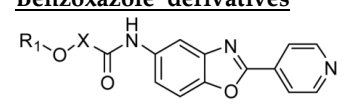

\begin{tabular}{lllll}
\hline Sl. No. & PubChem CID & X & R1 & Experimental pIC50 \\
1 & 71662080 & (R)-CHMe & 1-naphthyl & 8.92 \\
2 & 71297189 & (S)-CHMe & 1-naphthyl & 8.21 \\
3 & 71662081 & (S)-CHMe & $2,3-$ di-ClPh & 7.3 \\
4 & 71662082 & (S)-CHMe & $2-\mathrm{Cl}, 3-\mathrm{CF} 3 \mathrm{Ph}$ & 8.05 \\
5 & 71662258 & (S)-CHMe & 2-Cl,3-NO2Ph & 8.64 \\
6 & 71662259 & (S)-CHMe & $2,3-$ di-OMePh & 6.4 \\
\hline
\end{tabular}

\begin{tabular}{|c|c|c|c|c|c|c|c|c|c|}
\hline No & $\begin{array}{l}\text { PubChem } \\
\text { CID }\end{array}$ & Experimental pIC 50 & $\begin{array}{l}\text { Predicted } \\
\text { pIC }_{50}\end{array}$ & $\mathrm{BE}$ & IME & IE & TorE & VdwE & EE \\
\hline 1 & 2953497 & 7.36 & 7.66 & -9.03 & -10.22 & -1.38 & 1.19 & -10.29 & 0.07 \\
\hline 2 & 71661724 & 7.72 & 7.94 & -8.59 & -9.79 & -0.6 & 1.19 & -9.68 & -0.11 \\
\hline 3 & 71661725 & 6.98 & 7.29 & -8.61 & -9.8 & -1.58 & 1.19 & -9.8 & -0.01 \\
\hline 4 & 70850880 & 7.40 & 7.09 & -8.41 & -9.6 & -1.54 & 1.19 & -9.62 & 0.02 \\
\hline 5 & 71661894 & 7.55 & 7.66 & -8.51 & -10.0 & -1.15 & 1.49 & -9.92 & -0.07 \\
\hline 6 & 71661895 & 7.70 & 7.40 & -8.98 & -10.18 & -1.39 & 1.19 & -10.06 & -0.11 \\
\hline 7 & 46871958 & 8.52 & 8.15 & -8.97 & -10.16 & -1.1 & 1.19 & -10.06 & -0.11 \\
\hline 8 & 70851038 & 8.05 & 8.08 & -9.53 & -10.72 & -1.55 & 1.19 & -10.72 & 0.0 \\
\hline 9 & 71661897 & 7.57 & 7.95 & -9.69 & -11.18 & -2.1 & 1.49 & -11.1 & -0.08 \\
\hline 10 & 71662080 & 8.92 & 8.29 & -9.69 & -10.88 & -1.53 & 1.19 & -10.85 & -0.03 \\
\hline 11 & 71297189 & 8.21 & 8.06 & -9.72 & -10.92 & -1.43 & 1.19 & -10.92 & 0.01 \\
\hline 12 & 71662081 & 7.3 & 7.67 & -9.32 & -10.51 & -1.73 & 1.19 & -10.56 & 0.05 \\
\hline 13 & 71662082 & 8.05 & 7.69 & -8.82 & -10.32 & -0.76 & 1.49 & -10.24 & -0.08 \\
\hline 14 & 71662258 & 8.64 & 7.75 & -9.13 & -10.62 & -1.66 & 1.49 & -10.04 & -0.58 \\
\hline 15 & 71662259 & 6.4 & 6.16 & -8.17 & -9.96 & -2.47 & 1.79 & -10.0 & 0.04 \\
\hline
\end{tabular}

BE = Binding Energy; IME: Intermolecular Energy; IE = Internal Energy; TorE= Torsional; Energy; VdwE = vdW + Hbond + desolv Energy; EE= Electrostatic energy. 


\section{Results \& Discussion:}

Based on $R_{1}$ and $X$ groups at different positions, benzoxazole derivatives of $C$. parvum IMPDH were retrieved from literature [8] and are shown in Table $1 \& 2$. In docking studies of benzoxazole derivatives with IMPDH protein, best autodock score was used as criteria to interpret the best conformation among the 30 conformations, generated by AutoDock 4.2 program. The docking results of the benzoxazole derivatives with IMPDH protein were shown in Table 3. Further, the docked complexes were analyzed through Python Molecular Viewer software [15] for their interaction studies and were shown in Figure 3. Thus from the Complex scoring and binding ability it's deciphered that these compounds are promising inhibitors for IMPDH protein. MD simulation is a well-known theoretical technique and is mainly used for evaluating the stability of any predicted 3D model. Therefore, the constructed 3D model of protein-ligand complexes were processed for MD simulation for a 5 ps timescale with Langevin dynamics to control the kinetic energy, temperature, and/or pressure of the system. The RMSD values of complexes contain alpha carbon atoms, and all atoms were calculated by taking structure with reference conformation points. The RMSD values of complex versus time were shown in Figure 4. Relationship between experimental and predicted $\mathrm{pIC}_{50}$ values of Benzoxazole derivatives is shown in Figure 2.

\section{Conclusion:}

A QSAR model using pIC50 values for fifteen known benzoxazole derivatives binding with $C$. parvum IMPDH protein as dependent variable and molecular docking based predicted pIC50 with a correlation coefficient $\mathrm{r} 2$ is 0.7948 was reported.

\section{Acknowledgement:}

This article is a part of Ph.D. studies carried out by the author registered at IFTM University, Moradabad, India. The authors are grateful to Prof. R. M. Dubey, Vice Chancellor, IFTM University, Moradabad, India for providing an institutional research promotion grant and their generous help and encouragement to proceed with the studies in the School of Biotechnology IFTM University, Moradabad, India.

\section{References:}

[1] Rossle NF et al. Asian Pac J Trop Biomed. 2013 3: 916 [PMCID: PMC3793167]

[2] Xiao L \&, Ryan UM, Curr Opin Infect Dis. 2004 17: 483 [PMID: 15353969]

[3] Parsons MB et al. PLoS Negl Trop Dis. 2015 9: e0003529 [PMID: 25700265]

[4] Kotloff KL et al. Lancet 2013 382: 209 [PMID: 23680352]

[5] Sharling L et al. PLoS Negl Trop Dis. 2010 4: e794 [PMID: 20706578]

[6] Hedstrom L, Chem Rev. 2009 109: 2903 [PMID: 19480389]

[7] Pettersen EF et al. J Comput Chem. 2004 25: 1605 [PMID: 15264254]

[8] Gorla SK et al. J Med Chem. 2013 56: 4028 [PMID: 23668331]

[9] O'Boyle NM et al. J Cheminform. 2011 3: 33 [PMID: 21982300]

[10] HyperChem (TM) Release 7.5, Hypercube, Inc., 1115 NW 4th Street, Gainesville, Florida 32601, USA.

[11] Morris et al. J Computational Chemistry. 1998 19: 1639

[12] http://autodock.scripps.edu/resources/adt

[13] Phillips JC et al. J Comput Chem. 2005 26: 1781 [PMID: 16222654]

[14] Humphrey W et al. J Mol Graph. 1996 14: 33 [PMID: 8744570]

[15] Sanner MF, J Mol Graph Model. 1999 17: 57 [PMID: 10660911]

Edited by $P$ Kangueane Citation: Teotia et al. Bioinformation 12(3): 119-123 (2016) License statement: This is an Open Access article which permits unrestricted use, distribution, and reproduction in any medium, provided the original work is properly credited. This is distributed under the terms of the Creative Commons Attribution License. 